\title{
A Human TRPA1-Specific Pain Model
}

\author{
DStefan Heber, ${ }^{1}$ Markus Gold-Binder, ${ }^{1}{ }^{\circledR}$ Cosmin I. Ciotu, ${ }^{1}$ Martin Witek, ${ }^{2}$ @Nino Ninidze, ${ }^{3}$ Hans-Georg Kress, ${ }^{3}$ \\ and $\oplus^{-M i c h a e l ~ J . M . ~ F i s c h e r ~}{ }^{1}$ \\ Institutes of ${ }^{1}$ Physiology, ${ }^{2}$ Pharmacology, Center for Physiology and Pharmacology, and ${ }^{3}$ Division of Special Anesthesia and Pain Medicine, Department of \\ Anesthesia, Intensive Care and Pain Medicine, Medical University of Vienna, 1090 Vienna, Austria
}

The cation channel transient receptor potential ankyrin 1 (TRPA1) plays an important role in sensing potentially hazardous substances. However, TRPA1 species differences are substantial and limit translational research. TRPA1 agonists tested previously in humans also have other targets. Therefore, the sensation generated by isolated TRPA1 activation in humans is unknown. The availability of 2-chloro$N$-(4-(4-methoxyphenyl)thiazol-2-yl)- $N$-(3-methoxypropyl)-acetamide (JT010), a potent and specific TRPAl agonist, allowed us to explore this issue. To corroborate the specificity of JT010, it was investigated whether the TRPA1 antagonist (1E,3E)-1-(4-fluorophenyl)-2methyl-1-penten-3-one oxime (A-967079) abolishes JT010-elicited pain. Sixteen healthy volunteers of both sexes rated pain due to intraepidermal injections of different concentrations and combinations of the substances. The study design was a double-blind crossover study. All subjects received all types of injections, including a placebo without substances. Injections of the TRPA1 agonist dosedependently caused pain with a half-maximal effective concentration of $0.31 \mu \mathrm{M}$. Coinjection of A-967079 dose-dependently reduced and at a high concentration abolished JT010-induced pain. Quantification of JT010 by HPLC showed that a substantial part is adsorbed when in contact with polypropylene surfaces, but that this was overcome by handling in glass vials and injection using glass syringes. Isolated TRPA1 activation in humans causes pain. Thus, intradermal JT010 injection can serve as a tool to validate new TRPA1 antagonists concerning target engagement. More importantly, TRPA1-specific tools allow quantification of the TRPA1-dependent component in physiology and pathophysiology.

Key words: A-967079; JT010; pain model; psychophysics; target validation; TRPA1

\section{Significance Statement}

This study showed that activation of the ion channel transient receptor potential ankyrin 1 (TRPA1) alone indeed suffices to elicit pain in humans, independent of other receptors previously found to be involved in pain generation. The newly established TRPA1-specific pain model allows different applications. First, it can be tested whether diseases are associated with compromised or exaggerated TRPA1-dependent painful sensations in the skin. Second, it can be investigated whether a new, possibly systemically applied drug directed against TRPA1 engages its target in humans. Further, the general possibility of quantitative inhibition of TRPA1 allows identification of the TRPA1-dependent disease component, given that the substance reaches its target. This contributes to a better understanding of pathophysiology, can lay the basis for new therapeutic approaches, and can bridge the gap between preclinical research and clinical trials.

\section{Introduction}

The cation channel transient receptor potential ankyrin 1 (TRPA1) plays an important role in pain perception and is,

Received Dec. 3, 2018; revised Feb. 19, 2019; accepted Feb. 22, 2019.

Author contributions: S.H. and M.J.M.F. designed research; S.H., M.G.-B., C.I.C., M.W., and N.N. performed research; S.H. and M.J.M.F. analyzed data; S.H., H.-G.K., and M.J.M.F. wrote the paper.

M.J.M.F. received financial support by IZKF (Interdisciplinary Center for Clinical Research) Grant E27. We thank Birgit Schönfelder for experimental support and Dr. Christian Gruber for advice regarding the chemical analysis. We also thank Rok Blagus and Georg Heinze for support with the statistical planning (CeMSIIS, Medical University of Vienna).

The authors declare no competing financial interests.

Correspondence should be addressed to Stefan Heber at stefan.heber@meduniwien.ac.at.

https://doi.org/10.1523/JNEUROSCI.3048-18.2019

Copyright $@ 2019$ the authors among other potential applications, a promising target investigated for novel analgesics (Chen and Hackos, 2015). It is expressed in a substantial fraction of peripheral neurons (Nagata et al., 2005; Bautista et al., 2013; Usoskin et al., 2015; Li et al., 2016; da Silva Serra et al., 2016). TRPA1 acts as a sensor for irritating exogenous (Bandell et al., 2004; Bautista et al., 2006) as well as endogenous substances occurring during oxidative stress and inflammation (Andersson et al., 2008). For example, we found that the ion channel is activated by nitroxyl (Eberhardt et al., 2014) and methylglyoxal, which accumulates under diabetic conditions (Eberhardt et al., 2012). Furthermore, TRPA1 is also activated by a low pH (de la Roche et al., 2013), although this did not translate into a contribution of TRPA1 to the sensation of tissue acidosis in humans (Schwarz et al., 2017). 
In animal studies, TRPA1 acts as a pivotal sensor for potential tissue damage and thereby contributes to the perception of pain, representing a vital function of the nervous system (Obata et al., 2005). This includes inflammatory pain (e.g., in inflamed joints; Moilanen et al., 2015; Horváth et al., 2016) or visceral inflammation like colitis and pancreatitis (Mitrovic et al., 2010; Schwartz et al., 2013), but also neuropathic pain (Naik et al., 2006; Trevisan et al., 2016). Further, it plays a role in secondary inflammation after ischemia (Hamilton et al., 2016) and chemotherapy-induced peripheral neuropathic pain (Materazzi et al., 2012; Trevisan et al., 2013) or diabetic neuropathies (Koivisto et al., 2012).

However, a major drawback for pharmaceutical development are the substantial species-dependent differences in TRPA1 function. Major differences were found in response to pharmacological compounds, first described for menthol and in particular concerning noncovalent agonists (Xiao et al., 2008). Considerable differences have also been found between humans and distant species considering thermal stimuli (Cordero-Morales et al., 2011). Such differences were not found for example, for TRP cation channel subfamily V member 1 (TRPV1), and this could impede the TRPA1 drug discovery (Bianchi et al., 2012). Consequently, there is a need for human pain models on TRPA1 that help to bridge the translational gap between preclinical and clinical research.

Previously described chemical stimulation of TRPA1 in human subjects has used substances which are known to coactivate other targets. For example, allyl isothiocyanate (AITC) was recently used for a TRPA1-dependent pain model (Andersen et al., 2017); however, AITC can also activate TRPV1 (Gees et al., 2013). According to the reported $\mathrm{EC}_{50}$ of $0.65 \mathrm{nM}, 2$-chloro- $\mathrm{N}$-(4-(4methoxyphenyl)thiazol-2-yl)-N-(3-methoxypropyl)-acetamide (JT010) was the most potent commercially available agonist (Takaya et al., 2015). Further, it was shown to be specific regarding other pain-related TRP channels up to a concentration of $1 \mu \mathrm{M}$. By means of this substance, and the most potent commercially available antagonist (1E,3E)-1-(4-fluorophenyl)-2-methyl-1-penten-3-one oxime (A-967079), we established a human TRPA1-specific pain model. Here we show that an isolated TRPA1 activation caused pain in humans. This pain could be abolished by a TRPA 1 antagonist. The established pain model allows validation of new pharmacological antagonists and elucidation of the role of TRPA1 channels in human disease.

\section{Materials and Methods}

\section{Experimental design}

In a training session, conducted at least $1 \mathrm{~d}$ before the main trial, a subject was familiarized with the rating of pain by separate epidermal injection of control [50 $\mu \mathrm{l}$ phosphate-buffered synthetic interstitial fluid (PB-SIF)] and capsaicin $3.1 \mu \mathrm{M}(0.001 \%)$ as a positive control. This also allowed the subjects to decide on their further participation in the study.

The main trial had a double-blinded, single-group, randomized, placebo-controlled crossover design. Each of the 16 subjects (representing a single group) sequentially received nine injections that differed only in the concentrations of JT010 and A-967079 (Fig. 1). Both the experimenter and the volunteers were blinded regarding the injected substances. The sequences of the injected substances were predefined by a Latin-squares Williams design, which balances for first-order carryover effects (Williams, 1949). Sequences were generated in R by the "williams" program of the "crossdes" package. Volunteers were randomly allocated to these sequences.

Participants. Human volunteers of both sexes with full legal capacity between the ages of 19 and 41 years were recruited (inclusion age range, 18-70 years). Exclusion criteria were participation in another study within the last 4 weeks, medication intake (except contraception) or drug abuse, pregnancy or current breastfeeding, fever, allergic diseases, in particular asthmatic disorders and allergic skin diseases, any sensory deficit at the test site (volar forearm), medical history of angina pectoris, thrombosis, tumors, pneumonia, tuberculosis, bleeding diathesis, and diseases that could put the experimenter at risk (e.g., HIV, hepatitis).

Human psychophysical experiments. Experiments with human volunteers were approved by the ethics committee of the Medical University of Vienna (1799/2017) and were performed in accordance with the Declaration of Helsinki after written informed consent. Eight male and eight female subjects completed the study.

For experimental sessions, subjects were seated comfortably, and $50 \mu \mathrm{l}$ of each of the above-mentioned solutions were injected into the epidermal layer of the volar forearm using a glass syringe (100 $\mu$ l; Hamilton) with an attached cannula $(0.30 \times 12 \mathrm{~mm}$; Sterican, Braun $)$. The nine glass syringes were autoclaved after every use. Test sites were at least $5 \mathrm{~cm}$ apart and did not match the capsaicin injection site of the training session. There was a minimum period of $5 \mathrm{~min}$ between injections, which resulted in a duration of $\sim 45 \mathrm{~min}$ for the main trial. The right and left arm were used in alternating sequence for the injections. Pain was rated by means of a numeric rating scale (range, $0-1000$ for "no pain"; 100 for "maximum imaginable pain"). After insertion of the thin cannula, volunteers rated the resulting pain of this insertion by a single number (insertion pain). The solution was injected once the insertion pain had fully subsided. After each injection, subjects provided a pain rating every $5 \mathrm{~s}$ until six subsequent zero ratings were acquired. The area under the curve of pain (pain AUC) ratings was calculated as the sum of all pain ratings. This integral measure of pain after each injection was taken as the primary outcome variable. The primary hypothesis that the injection of 1 $\mu \mathrm{M}$ JT010 causes pain was tested by comparing the pain AUC values between the control injection (PB-SIF) and the injection containing $1 \mu \mathrm{M}$ JT010 (without A-967079). Second, we hypothesized that simultaneous coinjection of the TRPA1 antagonist A-967079 reduces pain induced by JT010. For this purpose, the injection containing $1 \mu \mathrm{M} \mathrm{JT010}$ and the one containing $1 \mu \mathrm{M}$ JT010 together with $10 \mu \mathrm{M}$ A-967079 were tested against each other.

Randomization, sequence generation, and blinding. The three pivotal injections for testing the two main hypotheses were placed at injection numbers 2, 4, and 6 in a balanced design to achieve a limited and defined time between these three injections as well as to avoid first-order carryover effects. The remaining six positions were also filled by Latin squaresbased sequences with a balanced design. Sequences were stored in identical closed envelopes. One envelope was randomly picked and discarded to define the sequence of the injected substances for each volunteer. To conceal the identity of prepared solutions both from the experimenter and the subjects, a third person prepared the solutions immediately before their experimental use and handed them to the blinded experimenter.

\section{Statistical analysis}

Based on a pilot experiment, which was also used to find an experimentally suitable JT010 concentration, we expected a pain AUC induced by 
JT010 $1 \mu \mathrm{M}$ of $\sim 400$ units. We assumed that the pain AUC in response to JT010 $1 \mu \mathrm{M}+$ A-967079 $10 \mu \mathrm{M}$ can only be in the range between the pain AUC in response to the control injection and the JT010 $1 \mu \mathrm{M}$ injection. Thus, the effect size concerning the secondary hypothesis was necessarily less or equal compared with the primary hypothesis, requiring an equal or greater sample size. Therefore, sample size estimation was based on the secondary hypothesis. We considered a pain AUC reduction by $10 \mu \mathrm{M}$ A- 967079 of $50 \%$ as relevant, corresponding to 200 units. Based on previous studies (Schwarz et al., 2017), we expected the SD of the differences of two injections in the same individual to be $\sim 220$ units. To detect a $50 \%$ reduction in pain AUC with a power of 0.8 , accepting a type I error probability of 0.05 and the assumed SD of differences, 12 volunteers were needed. We expected that up to $25 \%$ of all volunteers, who initially agreed to take part in this trial, could drop out after the session for familiarization. Therefore, we included a total of 16 volunteers, who all completed the main trial.

The primary statistical analysis was performed by a mixed linear model with pain AUC as a dependent variable and an identity covariance structure. The model included the subjects as levels of a random factor and "injection type" as fixed within-subject factor with nine levels according to the nine injections. To account for the crossover situation, the order of injections was included in the model as a second-degree polynomial. Planned contrasts were used to test predefined hypotheses: the primary hypothesis was tested by comparing the pain AUC values between the control injection (PB-SIF) and the injection containing $1 \mu \mathrm{M}$ JT010 (without A-967079). In case the primary null hypothesis had been rejected, the secondary hypothesis was tested by comparing the injections containing $1 \mu \mathrm{M} \mathrm{JT010} \mathrm{without} \mathrm{A-967079} \mathrm{and} \mathrm{the} \mathrm{one} \mathrm{containing} 1 \mu \mathrm{M}$ JT010 plus $10 \mu \mathrm{M}$ A-967079. Additional contrasts were performed in an exploratory manner without adjustment for multiple testing. Due to different variances between groups (heteroscedasticity), the main statistical analysis was performed with square-root-transformed pain AUC values. Unless stated otherwise, graphs show back-transformed leastsquares means (adjusted for the order of injections) with SEs on the original scale. To explore the effect of insertion pain on the pain AUC, it was added as a metric predictor to the primary mixed-linear model.

$\mathrm{EC}_{50}$ and $\mathrm{IC}_{50}$ values were estimated by fitting a four-parameter logistic dose-response curve to estimated means resulting from the mixed linear model. For combined presentation, in vivo and in vitro data were normalized by setting the maximum response to $100 \%$. The percentage of inhibition achieved by A-967079 $10 \mu \mathrm{M}$ was calculated for each individual separately using the following formula:

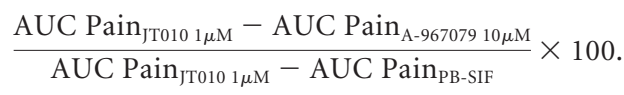

Thereafter the median percentage with its $95 \%$ confidence interval (CI) was determined by bias-corrected and accelerated bootstrapping with 1000 samples. The maximum pain rating and seconds until maximum pain, half-maximal pain, and time until pain has subsided were compared using a linear model with the three injections as withinsubjects factor. Statistical analyses were performed using IBM SPSS Statistics 24 and GraphPad Prism 7. Two-sided $p$ values $\leq 0.05$ were considered statistically significant. Results are reported as the mean \pm SEM, for datasets with skewed distribution median and 95\% confidence intervals are reported as indicated.

\section{Chemicals and solutions}

As in the previous study (Schwarz et al., 2017), a PB-SIF was used, in which all substances were dissolved. This is a modification of the synthetic interstitial fluid by Bretag (1969) in which hydrogen carbonate was replaced by dihydrogen phosphate, as described previously (Schwarz et al., 2017). The PB-SIF contains the following (in mM): sodium chloride 108 , potassium chloride 3.5 , disodium hydrogen phosphate 23 , magnesium sulfate 0.7 , calcium chloride 1.5 , sodium gluconate 9.6 , glucose 5.5 , and sucrose 7.6. The resulting pH 7.4 was controlled and titrated with sodium hydroxide or hydrogen chloride if necessary. Sterile solutions were obtained by using a $0.22 \mu \mathrm{m}$ syringe filter. PB-SIF was used as a control solution for all experiments and for diluting sterile stock solu- tions. JT010, A-967079, N-vanillylnonanamide, GSK1016790A, and pregnenolone sulfate were purchased from Sigma-Aldrich. Capsaicin was dissolved in ethanol $(10 \mathrm{~mm})$ and diluted to $3.1 \mu \mathrm{M}$ with PB-SIF before injection. Acetonitrile [ACN; high-performance liquid chromatography (HPLC) LC-MS grade] was obtained from VWR, formic acid $>98 \%$ was obtained from Applichem, methanol >99\% from Chem-Lab, and ultra-pure water was obtained from our Milli-Q plus system (Millipore).

\section{Special handling of JT010}

The volunteer study had to be performed twice due the special properties of JT010. Handling of the substance was adapted for the second round.

Psychophysics experiments round 1. All pipetting steps were performed in $1.5 \mathrm{ml}$ polypropylene tubes (Safe-Lock Tubes, Eppendorf) and with polypropylene pipette tips. A $10 \mathrm{~mm}$ stock solution was generated in DMSO, while further dilutions were then prepared in PB-SIF. Injections were performed with $0.3 \mathrm{ml}$ polypropylene syringes with a 27 gauge cannula (BD).

Psychophysics experiments round 2. The stock solution in DMSO was handled with polypropylene tips, while diluted solutions were maintained in glass vials and handled by means of Hamilton syringes. For both JT010 and A-967079, 10 mM stock solutions in DMSO were generated. From these, nine solutions containing 1000-fold the target concentrations of both substances were prepared in DMSO. Aliquots of $1.5 \mu$ l were stored in glass vials at $-20^{\circ} \mathrm{C}$ until use. Immediately before application into the epidermis of human subjects, $1498.5 \mu \mathrm{l}$ PB-SIF was added and the solution vortexed, leading to a final DMSO concentration of $0.1 \%$ in all injection solutions (including for the control solution without JT010). These solutions were not exposed to polypropylene tips or tubes during processing to avoid adsorption.

\section{Solid-phase extraction and chromatography analysis}

To investigate the loss of JT010 after contact with polypropylene surfaces, the substance was quantified by HPLC. JT010 was dissolved in deionized water and quantified using RP-C18 columns $(150 \times 3.0 \mathrm{~mm}, 2.5 \mu \mathrm{M}$; Kinetex, Phenomenex), in a Dionex Ultimate 3000 UHPLC focused System. The mobile phase composed of solvent A ( $100 \%$ water containing $0.05 \%$ trifluoroacetic acid) and solvent B [water/acetonitrile (10:90, v/v) containing $0.05 \%$ trifluoroacetic acid) was used with a flow rate of 0.3 $\mathrm{ml} / \mathrm{min}$. The gradient used started at $50 \%$ solvent B and was linearly raised to $100 \%$ solvent $\mathrm{B}$ within $10 \mathrm{~min}$, which was continued for a further $5 \mathrm{~min}$. Afterward, the column was re-equilibrated to $50 \%$ solvent A in $5 \mathrm{~min}$. Absorption was monitored for 215, 254, 280, and $320 \mathrm{~nm}$.

\section{HPLC with subsequent mass spectroscopy}

An ACQUITY Arc chromatographic system was used, consisting of a Sample Manager FTN-R, a Quaternary Solvent Manager-R, a column oven, an ACQUITY QDa, a Single Quadrupole Mass Spectrometer, and MassLynx V4.1 software (Waters). The mobile phase consisted of 50\% water, $50 \%$ acetonitrile, and $0.1 \%$ formic acid, and was mixed by the solvent manager at isocratic conditions with a flow rate of $0.5 \mathrm{ml} / \mathrm{min}$. JT010 $2 \mu \mathrm{M}$ was dissolved in the mobile phase. The Luna C18 $2.5 \mu \mathrm{m}$ HST $50 * 2 \mathrm{~mm}$ (Phenomenex) column was heated to $40^{\circ} \mathrm{C}$; the autosampler was adjusted to $25^{\circ} \mathrm{C}$. Data were acquired in single ion record mode, and total ion count in scan mode. Ionization was performed with electrospray ionization (ESI) in positive and negative mode. For both single ion record and total ion count, the capillary voltage was $0.8 \mathrm{kV}$ and the cone voltage was $15 \mathrm{~V}$. The probe temperature was $600^{\circ} \mathrm{C}$, and the scan range was from 200 to $1000 \mathrm{~m} / z$ (mass/charge ratio) For single ion record the $\mathrm{m} / \mathrm{z}$ was adjusted to 355 in ESI-positive mode for JT010. Mass distribution over the whole peak chromatographic output was evaluated. All measurements were performed at least fivefold.

\section{Intracellular calcium measurements}

CHO cells stably expressing human TRPAl were provided by Stuart Bevan (King's College London, London, UK). HEK293t cells were transfected with plasmids using jetPEI transfection reagent (Polyplus). The human TRPA1 plasmid was obtained from Ardem Patapoutian (Scripps Research, La Jolla, CA), hTRPA1 was mutated and subcloned to human TRPA1-C621S-C641S-C665S (Meents et al., 2016), human TRPV1 was 

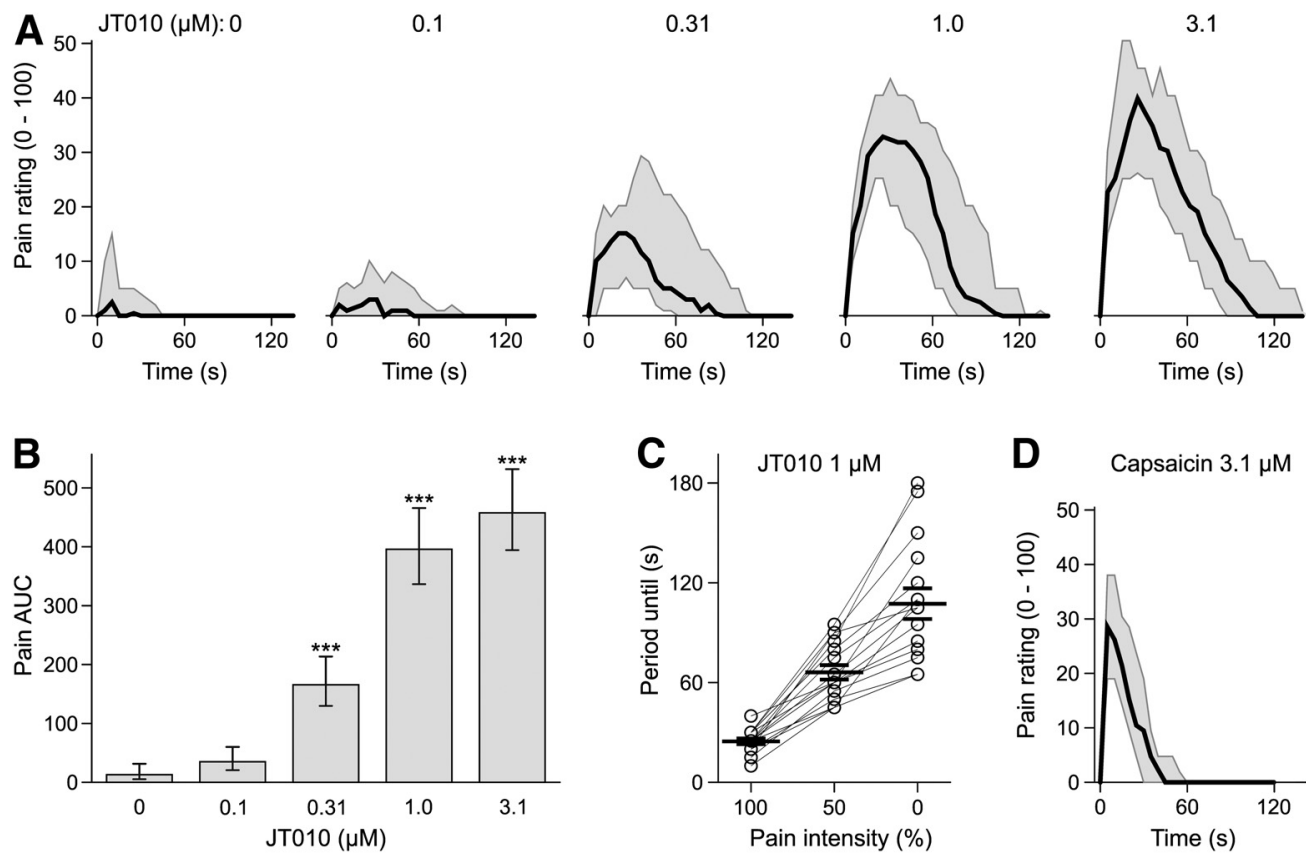

Figure 2. JT010 injection causes pain in human subjects. A, JT010 dissolved in $50 \mu \mathrm{IPB}$-SIF was injected intradermally into the volar forearm. Pain ratings are visualized with median and $95 \%$ confidence interval. B, JT010 induced a concentration-dependent increase in the pain AUC. C, Pain ratings increased rapidly and reached a maximum (100\%) $25 \mathrm{~s}$ after injection, halved (50\%) after $66 \mathrm{~s}$, and disappeared (0\%) after $108 \mathrm{~s}$ (mean values). $\boldsymbol{D}$, Median pain ratings with $95 \%$ confidence intervals after capsaicin injections. ${ }^{* * *} p<0.001$.

obtained from Carla Nau (Universitätsklinikum Schleswig-Holstein, Lübeck, Germany; Leffler et al., 2008), human TRPV4 was obtained from Xuming Zhang (Aston University, Birmingham, UK), human TRPM3 was obtained from Johannes Oberwinkler (Center for Mind, Brain and Behavior, Marburg, Germany), and human TRPM8 was obtained from Thomas Voets (KU Leuven, Leuven, Belgium). Cells were grown in 96well black walled plates. Cells were loaded with Calcium 6 for $2 \mathrm{~h}(\mathrm{Cal}-$ cium 6 Kit, Molecular Devices) in a physiological solution, containing the following (in mM): $145 \mathrm{NaCl}, 5 \mathrm{KCl}, 10$ glucose, 10 HEPES, 1.25 $\mathrm{CaCl}_{2}$, and $1 \mathrm{MgCl}_{2}$, buffered to $\mathrm{pH} 7.4$ with $\mathrm{NaOH}$. According to the manufacturer protocol, cells were not washed as the extracellular dye was chemically quenched. Calcium 6 fluorescence excited at $488 \mathrm{~nm}$ every $2.5 \mathrm{~s}$ served as an index of intracellular calcium. Assays were performed at $37^{\circ} \mathrm{C}$ in a FlexStation 3 (Molecular Devices). A volume of $50 \mu \mathrm{l}$ was pipetted automatically according to a preset protocol into $100 \mu \mathrm{l}$ of medium in the wells. Fluorescence change relative to baseline fluorescence is reported $\left(d F / F_{0}\right)$.

\section{Results}

\section{TRPA1 activation in human skin causes pain}

Psychophysical experiments including intradermal JT010 injections showed that pain AUC generally differed among all nine injections (mixed linear model, main effect of factor "injection type": $\left.F_{(8,118)}=36.57, p<0.001\right)$. The subsequently estimated contrast corresponding to the main hypothesis showed that an injection containing $1 \mu \mathrm{M}$ JT010 causes more pain than a control injection (planned contrast: $t_{(118)}=10.4, p<0.001$; Fig. $2 A, B$ ). In addition, we explored the effects of different concentrations, which revealed that both 0.31 and $3.1 \mu \mathrm{M}$ JT010 significantly caused pain ( $p<0.001$ each, tested against control injection). Notably, a cubic polynomial contrast indicated that the increase of pain due to rising JT010 concentrations significantly levels off at higher JT010 concentrations (cubic trend: $t_{(118)}=-2.7, p=0.008$ ).

Further characterization of this human pain model showed that maximal pain ratings occur after $\sim 30 \mathrm{~s}$, pain ratings halve within $\sim 70 \mathrm{~s}$, and pain subsides within $120 \mathrm{~s}$ (Fig. $2 \mathrm{C}$ ). The peak pain after JT010 injection occurs significantly later (median time
25 s) than after the injection of a comparable concentration of capsaicin (median time, $5 \mathrm{~s}$ ), which was used for familiarization with the pain rating (Wilcoxon signed rank test: $z=3.46, p<$ $0.001)$

Ratings of pain due to insertion of the cannula were not different between the various solutions (Fig. 3A). To investigate to what extent the sensitivity of each injection spot, reflected by the cannula insertion pain, might have affected the results, insertion pain was added as covariate in the primary statistical model. There was a positive relationship between insertion pain and pain AUC $\left(t_{(127)}=2.51, p=0.013\right)$; however, this adjustment did not change the estimated pain ratings to a relevant extent (Fig. $3 B$ ).

The quality of pain during the whole set of injections was interrogated by the German version of the Pain Perception Scale (Geissner, 1995); both after the capsaicin injection during the training session and after the main trail. The German version of the Pain Perception Scale was used as all subjects were German native speakers. No relevant differences were found between JT010 and capsaicin (Fig. 4). No volunteer reported a cold sensation.

\section{Discrepancy between the previously reported and observed $\mathrm{EC}_{50}$ of JT010}

JT010 was the TRPA1 agonist of choice for this study due to its reported specificity and low $\mathrm{EC}_{50}$ of $0.65 \mathrm{~nm}$. Surprisingly, the estimated $\mathrm{EC}_{50}$ of JT010 based on pain ratings was $308 \mathrm{~nm}$ (Fig. $5 A$ ). Thus, a cell-based assay was used. In a closely spaced concentration range and 12-fold measurements from 3 separate days, the result was an $\mathrm{EC}_{50}$ of $47 \mathrm{nM}$, which is lower compared with the estimated $308 \mathrm{~nm}$ in psychophysical experiments.

A potential temperature dependence and cell line dependence of the $\mathrm{EC}_{50}$ was addressed by repetition of the calcium assays in hTRPA1-expressing HEK293t cells. Activating JT010 concentrations induced a $29 \%$ higher calcium influx at $25^{\circ} \mathrm{C}$ compared with $37^{\circ} \mathrm{C}$ lower temperatures $\left(F_{(1,91)}=15.6, p<0.001\right.$, extra sum-of-squares $F$ test), but the $\mathrm{EC}_{50}$ was not shifted $\left(F_{(1,91)}=\right.$ 

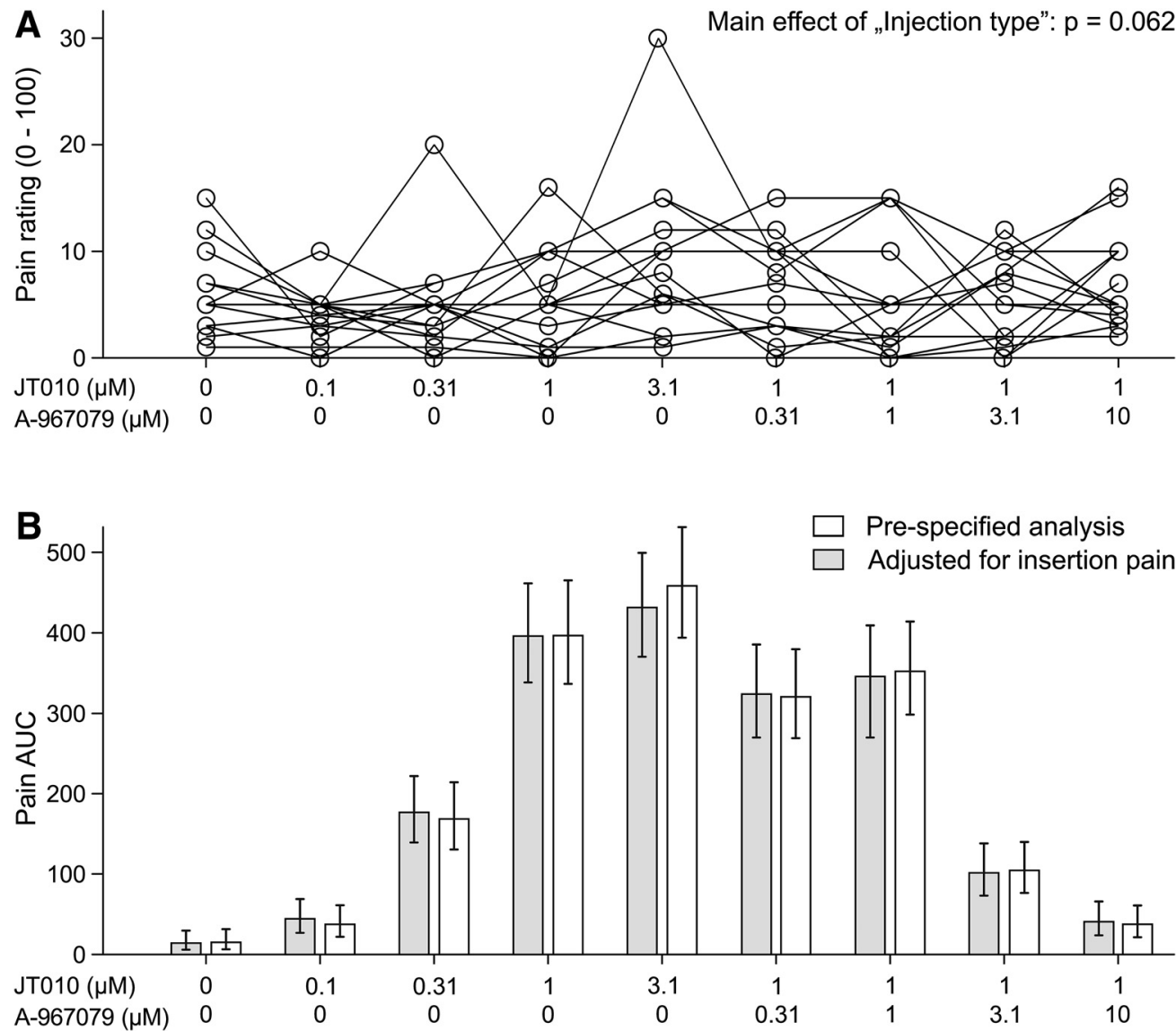

Figure 3. Maximal pain ratings due to insertion of the cannula before injection. $\boldsymbol{A}$, Individual maximal pain ratings due to cannula insertion before injection. Pain ratings elicited by insertion of the cannula did not differ between injection types. $\boldsymbol{B}$, Estimated geometric pain AUC means without (prespecified analysis) and with adjustment for differences in insertion pain. Statistical adjustment of pain AUC values for insertion pain did not alter estimated pain AUC values to a relevant extent, showing that the injection spot did not affect the primary results to a relevant extent.

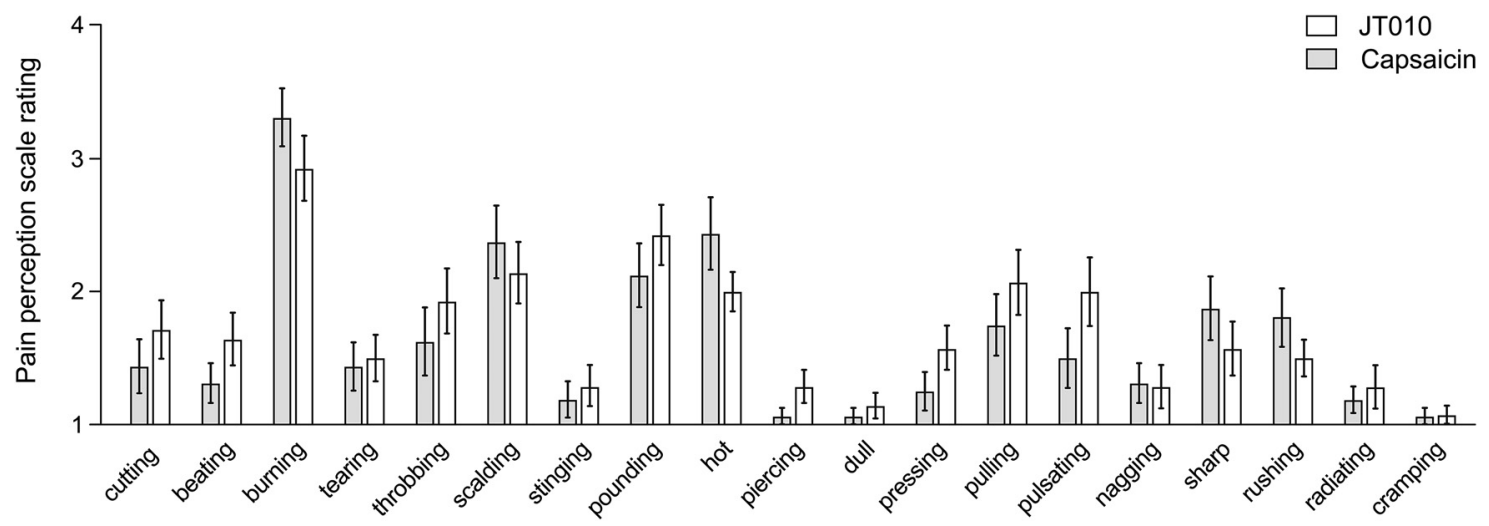

Figure 4. Pain perception of capsaicin and JT010. The perception was interrogated by the Pain Perception Score. Most volunteers rated affective parameters 1-14 with "1," corresponding to "not applicable" for both substances (data not shown). The sensory characteristics (parameters, 15-33) of capsaicin and JT010 appear largely identical. It needs to be mentioned that these ratings are from separate sessions and the study was not powered to investigate this exploratory question.

0.07, $p=0.79$ ). However, the published $\mathrm{EC}_{50}$ of $0.65 \mathrm{~nm}$ was based on endpoint reads. Representative calcium traces induced by the application of 100 and $3162 \mathrm{~nm} \mathrm{JT010} \mathrm{showed} \mathrm{that,} \mathrm{despite}$ pronounced differences of calcium traces, the endpoints can be quite similar. In contrast, the area under the first $30 \mathrm{~s}$ of the curve of calcium signals are a suitable measure to quantify calcium influx and show clear differences between JT010 concentrations (Fig. 5B). Therefore, in this manuscript the area under the curve of calcium signal was used for $\mathrm{EC}_{50}$ estimation. An exposure of the cysteine mutant hTRPA1-C621S-C641S-C665S to JT010 1 $\mu \mathrm{M}$ and $3.1 \mu \mathrm{M}$ shows at best a minimal response compared with the wild-type hTRPA1 ( $n=2$ each; Fig. $5 A$ ), confirming a covalent mode of action. As specificity of JT010 was previously only shown up to $1 \mu \mathrm{M}$, we further extended this considering human TRPV1. JT010 $1 \mu \mathrm{M}$ induced 1.2\%, $3.1 \mu \mathrm{M} 2.8 \%$ and $10 \mu \mathrm{M} 5.6 \%$ of the response to capsaicin $2 \mu \mathrm{M}(n=2$ each, data not shown). JT010 1-10 $\mu \mathrm{M}$ did not activate human TRPV4 and human TRPM3 expressed in HEK293t cells, which could be activated by 
reference agonists GSK1016790A and pregnenolone sulfate. Also, JT010 $10 \mu \mathrm{M}$ did not activate TRPM8.

\section{JT010 induced pain was abolished by coapplication of the TRPA1 antagonist A-967079}

As expected, coapplied A-967079 $10 \mu \mathrm{M}$ inhibited pain induced by JT010 $1 \mu \mathrm{M}$ (planned contrast corresponding to secondary hypothesis: $t_{(118)}=8.66, p<$ 0.001 ; Fig. $6 A, B)$. The results showed a pronounced, concentration-dependent reduction of pain. Pain induced by $1 \mu \mathrm{M}$ JT010 could be reduced by $98 \%$ (median) due to coapplication of $10 \mu \mathrm{M}$ A-967079. The bootstrapped $95 \%$ confidence interval ranges from $96 \%$ to $101 \%$; therefore, the results are consistent with complete inhibition (Fig. 6C). Exploratory analyses of other concentrations showed that the pain AUC was not significantly reduced by 0.31 and $1 \mu \mathrm{M} \mathrm{A}-967079$, respectively. Interestingly, maximal pain ratings in response to these injections were markedly lower than in response to $1 \mu \mathrm{M} \mathrm{JT010}$ alone (Fig. 6D), whereas the time course of pain ratings was delayed (Fig. $6 E$ ). The estimated $\mathrm{IC}_{50}$ of A-967079 based on pain AUC was $2.1 \mu \mathrm{M}$ (Fig. 7).

\section{Results of the first trial attempt}

The data presented above represent the second conduction of the trial and were generated with optimized handling of JT010. In a first round, induced pain ratings were substantially lower, and only the injections that were intended to contain JT010 3.1 $\mu \mathrm{M}$ caused more pain than the control injection. Contrary to our expectations, an intended concentration of JT010 1 $\mu \mathrm{M}$ did not cause pain. (Fig. $8 A$ ).

Subsequently, the reason for such low pain ratings was investigated, and as a result JT010 handling was optimized as described below. This led to a reapproval of a second round by the institutional ethics committee, in which pain ratings were substantially higher. Based on these pain ratings, the actual concentration in solutions with an intended JT010 concentration of $3.1 \mu \mathrm{M}$ in the first round was estimated to have been only $0.42 \mu \mathrm{M}$, corresponding to a substance loss of $86 \%$ (Fig. $8 B$ ).

\section{JT010 contact to polypropylene should be avoided}

After conducting the first round of psychophysical experiments, it was reasoned that the substance could have been adsorbed and/or chemically modified by polypropylene tubes and pipette tips. To further address this issue, aqueous JT010 solutions, prepared in glass vials, were put into polypropylene tubes, whereupon the JT010 concentration was again quantified by HPLC. Results showed that the concentration was reduced to $67 \pm 1 \%$ after a single polypropylene contact. To differentiate between adsorption and chemical reaction, the lipophilic solvent acetonitrile was used to detach JT010 adhering to polypropylene tubes. Acetonitrile recovered $23 \pm 1 \%$ of the initial JT010 amount from the polypropylene tube in chemically unaltered form. In sum, the fate of $90 \%$ of the initial JT010 amount could be clarified (Fig. $9 A, B)$

Next, the loss of JT010 due to tubes and tip contact was investigated. Repetitive HPLC from the same glass vials as control shows that a JT010 $10 \mu \mathrm{M}$ concentration remains nearly constant over $3 \mathrm{~h}$ in glass vials without polypropylene contact. To explore how much substance is lost due to polypropylene tip contact, a second group of JT010 solutions, stored in glass vials, was exposed twice to the same polypropylene pipette between measurements. In a third group, probes were pipetted into polypropylene tubes using polypropylene tips and vortexed, whereupon the solution was transferred after $5 \mathrm{~min}$ with the same pipette tip back into the same glass vial, corresponding to two pipette tip contacts and an additional polypropylene tube contact (Fig. 9C). Subtraction of the loss observed in group two from the loss observed in group three allowed to estimate the loss by polypropylene tube contact alone. A two-way ANCOVA with baseline values as covariate, time as a within-subjects factor, and group as a betweensubjects factor showed that the percentage change of JT010 concentration from the respective previous measurement was not different for each time point ( $n=3$ /group; time main effect, $p=0.3$; time $\times$ group interaction, $p=0.5)$, but differed among the three groups (group main effect, $p<0.001$ ). After every hour in a glass vial, the measured JT010 concentration was $0.8 \pm 0.3 \%$ lower. Every double contact to polypropylene tips reduced the 


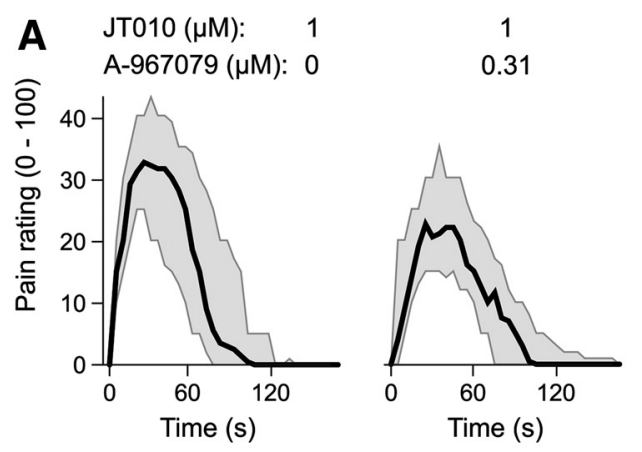

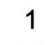
1
1
1.0
3.1
10
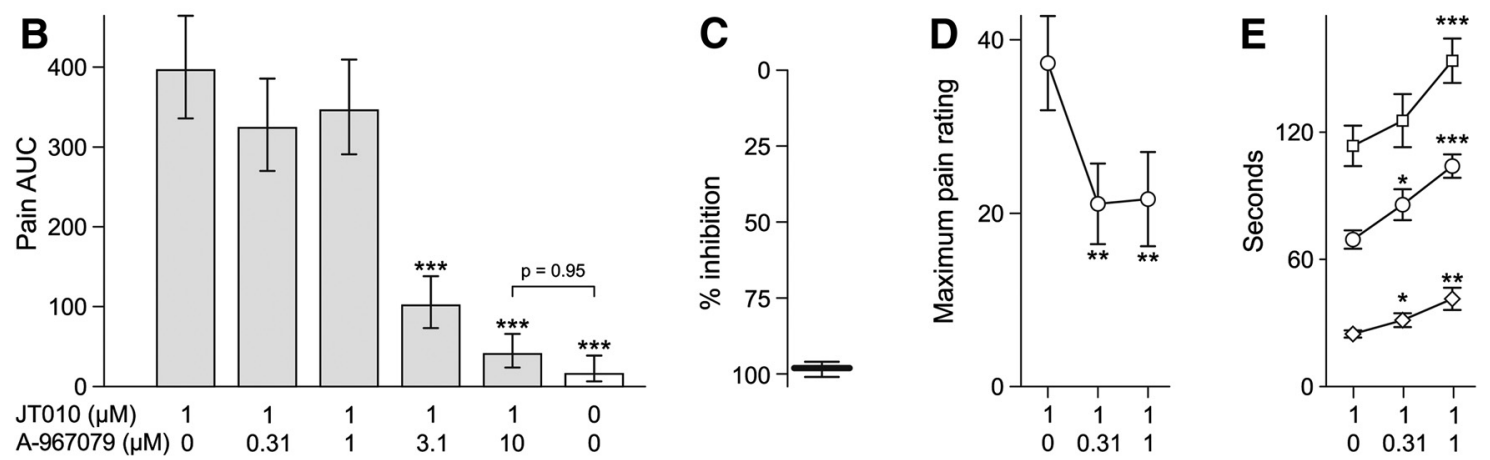

Figure 6. JT010-induced pain is abolished by A-967079 in healthy human subjects. A, A-967079 was coinjected with JT010 $1 \mu \mathrm{m}$ intradermally in $50 \mu$ linto the volar forearm. Pain ratings are presented as the median with $95 \%$ confidence interval. B, A-967079 inhibited the pain AUC, the highest concentration was not different from a control injection without JT010.C, A-967079 $10 \mu \mathrm{M}$ median inhibition of JT010-induced pain was estimated to be $98 \%$ (visualized with $95 \% \mathrm{Cl}$, which includes $100 \%$ inhibition). D, E, Although the lower concentrations of A-967079 did not reduce the pain AUC, the maximum pain rating was lower $(\boldsymbol{D})$, and the time course of reported pain was more delayed $(\boldsymbol{E})$. This applies to the time of maximum pain (diamond), the time of half-maximal pain (circle), and the time when pain subsided (square). ${ }^{*} p<0.05,{ }^{* *} p<0.01$ and ${ }^{* * *} p<0.001$.

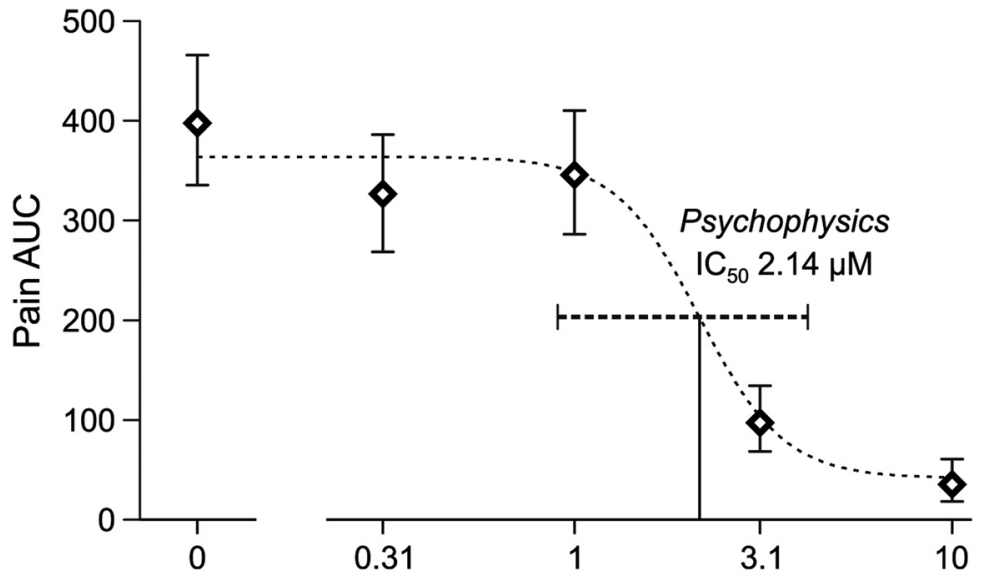

Figure 7. $\mathrm{IC}_{50}$ of $\mathrm{A}-967079$ in the human pain model. Based on the area under the curve of pain ratings after injection, the $\mathrm{IC}_{50}$ value and the respective $95 \%$ confidence interval were estimated.

amount of remaining JT010 by $11.2 \pm 0.4 \%$ (first vs second group, Sidak post hoc test, $p<0.001$ ), every contact to polypropylene tubes reduced this further by $16.1 \pm 0.4 \%$ (second vs third group, Sidak post hoc test, $p<0.001$ ). Each tube contact including pipetting resulted in a reduction by $27.3 \pm 0.4 \%$ (first vs third group, Sidak post hoc test, $p<0.001$ ).

It was hypothesized that substance loss due to adherence and/or chemical modification is reduced if JT010 is dissolved in a more lipophilic solvent, in particular DMSO. Hence, a defined amount of JT010 was either dissolved in $\mathrm{H}_{2} \mathrm{O}$ or DMSO in glass vials $(n=5$ each). Subsequently, each probe was pipetted with polypropylene tips into polypropylene tubes, out of which the solutions were removed again with polypropylene tips into glass vials. This procedure was repeated a second time. The elution from the chromatography column showed a peak at $2.5 \mathrm{~min}$, which was absent in blank controls (Fig. 9D). The mass spectrum of this peak showed a mass of $355 \mathrm{Da}$, which coincides with the published mass of JT010 (Fig. 9E). To quantify JT010 loss in $\mathrm{H}_{2} \mathrm{O}$ or DMSO, measurements were repeated by exclusively recording ions with a mass of $355 \mathrm{Da}$ (single ion record). Results showed that the area under the JT010 peak is diminished to a greater extent due to polypropylene contact in $\mathrm{H}_{2} \mathrm{O}$ compared with DMSO as the solvent (two-way ANOVA with time as a within-subject factor and solvent as a between-subject factor; time $\times$ solvent interaction, $p<0.001$; difference between $\mathrm{H}_{2} \mathrm{O}$ and DMSO after one and two tip contacts, $p<0.001$ each; Fig. $9 F, G)$. The scan mode with positive and negative ionization revealed further peaks. However, these also occurred in solutions with contact to polypropylene only, including JT010-free controls, and can therefore be considered a result of desorption from the "clean" reaction tubes. The results do not support new chemical derivatives arising from JT010, at least not exceeding a marginal level. Based on the observed adsorption, handling of JT010 solutions was adjusted to minimize polypropylene contact for aqueous JT010 solutions. This was achieved by using glass HPLC vials and Hamilton glass syringes. 

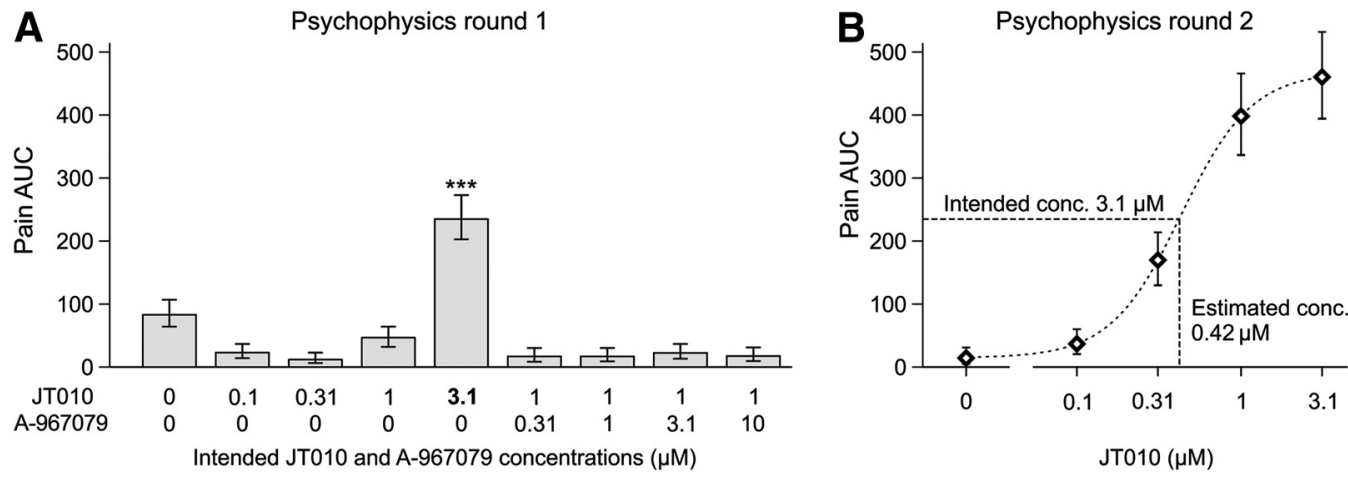

Figure 8. Results of the first round of psychophysical experiments. $A$, Pain AUC values with conventional substance handling using polypropylene materials were lower than expected. $\boldsymbol{B}$, Based on the concentration-response curve of the second round of experiments with optimized JT010 handling without polypropylene contact, the observed response to an intended concentration of 3.1 $\mu \mathrm{m}$ only equals to what can be expected from $0.42 \mu \mathrm{m}$ remaining substance. ${ }^{* * *} p<0.001$.
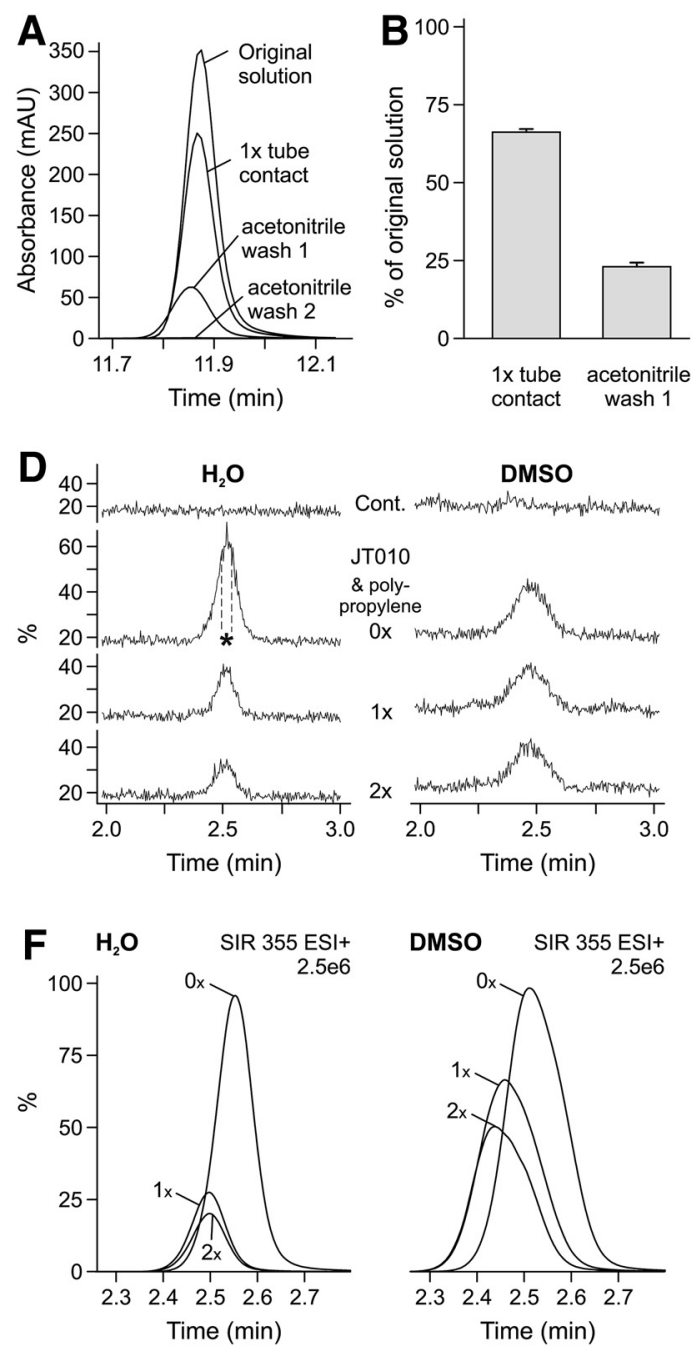
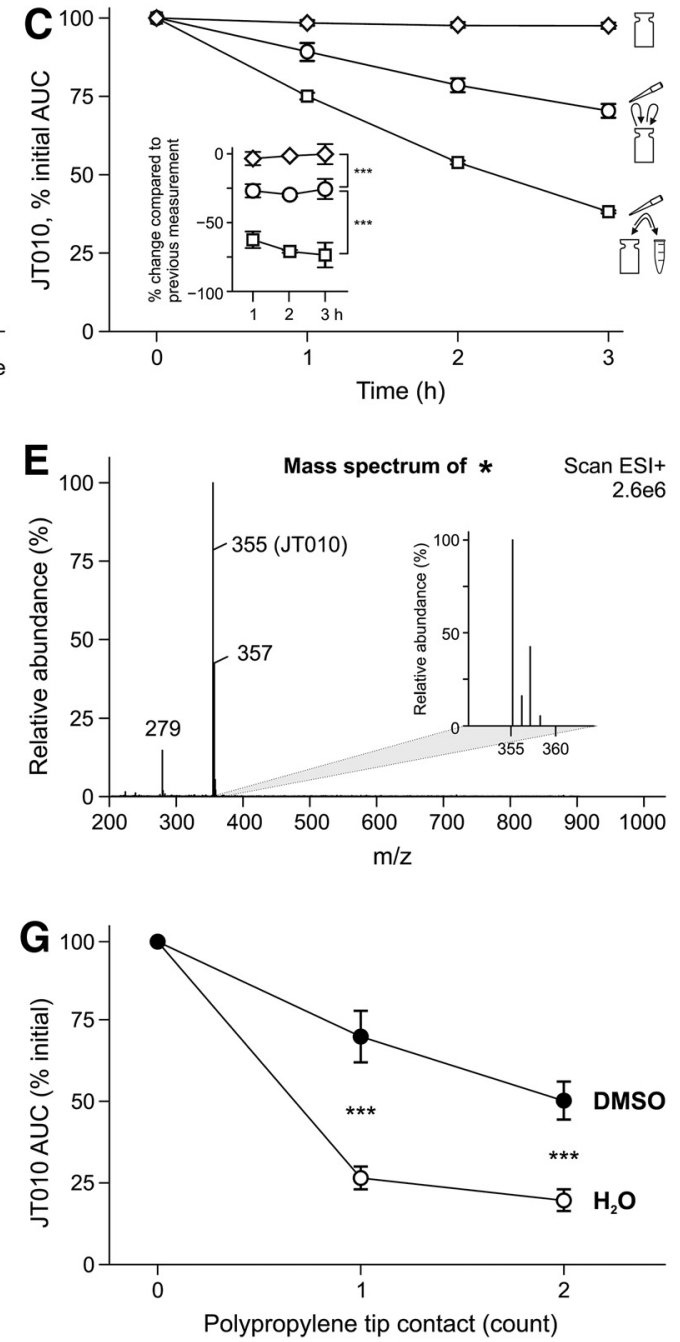

Figure 9. JT010 requires special handling. $A$, To identify sources of substance loss, JT010 $10 \mu \mathrm{m}$ in a glass vial was measured by HPLC (original solution). The substance was poured into a polypropylene reaction tube $(1 \times$ tube contact). JT010 could be recovered by an acetonitrile wash, but a second wash with acetonitrile did not recover further JT010. $\boldsymbol{B}$, Threefold repetition shows $67 \%$ of JT010 in solution, and $23 \%$ could be desorbed by acetonitrile. C, Repetitive HPLC of JT010 $10 \mu \mathrm{m}$ from the same glass vial (diamonds), after contact with the same polypropylene pipette tip twice between measurements (circles), and after transfer into a polypropylene tube and back into the same glass vial by the same polypropylene tip after 5 min (squares). Every contact to polypropylene caused a substantial substance loss. D, Representative HPLC protocol that detected the injected JT010 $2 \mu \mathrm{m}$ at $\sim 2.5$ min, which was reduced by polypropylene contacts $(0-2 \times)$ and absent in JT010-free controls (Cont.). $\boldsymbol{E}$, The mass spectrum covering the period at $\sim 2.5$ min shows a predominant peak of $355 \mathrm{~m} / z$ as expected for JT010. The mass spectrum shown in $\boldsymbol{E}$ refers to the period marked with ${ }^{*}$ in $\boldsymbol{D}$. $\boldsymbol{F}$, The single ion record (SIR) of $355 \mathrm{~m} / z$, averaged from five runs $\left(100 \%=2.5 \times 10^{6}\right.$ counts) in water and DMSO. G, Area under the curve recorded for JT010 $2 \mu \mathrm{m}$ due to one and two polypropylene tip contacts in water and DMSO. ${ }^{* * *} p<0.001$. 


\section{Discussion}

We demonstrated that an isolated and specific stimulation of TRPA1 in human skin causes pain. The established TRPA1specific pain model is based on the potent TRPA1 agonist JT010. Specificity of the easily implementable human pain model for TRPA1 is further corroborated by complete inhibition by the TRPA1 antagonist A-967079. The model can be used to validate drug candidates inhibiting TRPA1 in humans.

Substantial species differences, in particular between mice and humans, hamper translation of preclinical to clinical results regarding TRPA1 function (Xiao et al., 2008; Cordero-Morales et al., 2011). Previous studies of TRPA1-related human pain models investigated at best TRPA1 among other targets, mainly due to the lack of a selective agonist, where specificity can hardly be claimed. AITC, for instance, was injected in 1-9 M (10-90\%) concentration in psychophysical experiments (Andersen et al., 2017). Concentrations $>100 \mu \mathrm{M}$, however, were found to activate TRPV1 as well, and at higher concentrations in the millimolar range the activation of TRPV1 exceeds TRPA1 by many times, as the latter can even be inhibited again (Everaerts et al., 2011; Gees et al., 2013; Meseguer et al., 2014). For example, when AITC $10 \mathrm{~mm}$ was injected less than half of the behavioral responses was blocked by TRPA1 inhibition (Sałat and Filipek, 2015).

Similarly, cinnamaldehyde was applied topically in a concentration of $\geq 75 \mathrm{~mm}$ (Roberts et al., 2011), and a concentration as low as $5 \mathrm{~mm}$ was demonstrated to activate TRPV3 (Macpherson et al., 2006). Recently, we injected human subjects with carvacrol, which is a noncovalent agonist occurring naturally in oregano and thyme. TRPA1 antagonist A-967079 only partially (31\%) inhibited the carvacrol-induced pain (Schwarz et al., 2017), which might be explained by carvacrol $500 \mu \mathrm{M}$ also targeting anoctamin 1 (Takayama et al., 2017). For many other substances, for example, 15- $\delta \mathrm{PGJ}_{2}$ (Cruz-Orengo et al., 2008), a TRPA1specific mechanism has been reported in animals, but no translation to a human pain model has been attempted.

\section{JT010-induced pain}

The specificity of JT010 $1 \mu \mathrm{M}$ for TRPA1 was established by testing a panel of TRP channels involved in nociception, including TRPV1, TRPV3, TRPV4, TRPM2, TRPM8, and TRPC5, using a concentration of $1 \mu \mathrm{M}$ (Takaya et al., 2015). To allow for estimation of the $\mathrm{EC}_{50}$, concentrations of JT010 included $3.1 \mu \mathrm{M}$. Therefore, lack of activation of TRPV1 expressed in HEK293t cells by JT010 $3.1 \mu \mathrm{M}$ (and $10 \mu \mathrm{M}$ ) has been demonstrated in this manuscript. The significant cubic polynomial contrast shows that at lower concentrations an increase of JT010 pain was associated with increased pain, but this levels off at higher JT010 concentrations. This might be indicative that a concentration of $3.1 \mu \mathrm{M}$ JT010 is above the $\mathrm{EC}_{50}$, allowing the estimation of the latter.

Capsaicin was used as a test stimulus in a training session in all subjects. As observed in cellular experiments, the onset of pain was faster for capsaicin compared with JT010. This is probably explained by the slower chemical activation, including covalent modification of TRPA1 (Hinman et al., 2006; Macpherson et al., 2007; Bahia et al., 2016). The intradermal injection model caused a short pain sensation, which showed good tolerability in volunteers. This is also supported by completion of the study by all recruited subjects.

In our study, the pain perception profile of capsaicin and JT010 was largely identical, generating a primarily burning pain sensation. TRPA1 was originally proposed to be a noxious cold sensor, a notion that turned out controversial. The human subjects in the present study did not report cold or hot perception.
Our results suggest that it cannot be excluded that TRPA1 contributes to cold perception, but an isolated TRPA1 activation is at least not sufficient to generate cold pain.

\section{JT010 stability}

Compared with pilot experiments for protocol optimization, during the first run of the study all samples were generated at one point in time simultaneously, and included several contacts to polypropylene surfaces. In addition, preparation, including blinding, took substantially longer, which might further explain the estimated loss in actually injected JT010 concentration. Adsorption, shown by substance recovery after a single exposure and by deadsorption with a lipophilic solvent, is a general problem of lipophilic compounds, and therefore may apply to other TRPA1 agonists. We observed a surface-dependent adsorption of a considerable amount of JT010. This can be expected to be concentration dependent, with a higher fraction being adsorbed to polypropylene for lower substance concentrations. Without investigating this in detail, comparison of the higher loss of JT010 2 $\mu \mathrm{M}$ compared with $10 \mu \mathrm{M}$ supports this assumption (Fig. 9, compare $G, C)$. The results do not support a chemical instability of JT010 in aqueous solution during experimentally relevant periods of time.

Several available pipette tips designed to handle lipophilic chemicals were tested and in all of these a considerable JT010 loss was observed (data not shown). Accordingly, the study suggests the handling of stocks in a lipophilic solvent and the avoiding of contact of the diluted solutions with plastic surfaces.

\section{$\mathrm{EC}_{50}$ considerations}

The $\mathrm{EC}_{50}$ from human psychophysics was higher than what might have been expected. Therefore, concentration-response curves were measured in vitro in $\mathrm{CHO}$ cells with stable TRPA1 expression. The automated and heated pipetting fluorescence plate reader allowed using small concentration steps over a large concentration range at $37^{\circ} \mathrm{C}$. All measurements were performed 12 -fold, on 3 separate experimental days and were consistent across experimental days. The $\mathrm{EC}_{50}$ in cells was $47 \mathrm{~nm}$ at $37^{\circ} \mathrm{C}$, which is substantially higher than reported previously. This was supported by a separate experiment on hTRPA1-expressing HEK293t cells, where a higher calcium influx was observed at lower temperature, a behavior fitting cold facilitation and opposed to heat-activated phenotypes. Unexpectedly, the $\mathrm{EC}_{50}$ was not shifted between experiments at $25^{\circ} \mathrm{C}$ and $37^{\circ} \mathrm{C}$. In summary, recording temperature does not explain the observed differences in $\mathrm{EC}_{50}$ between the present data and those reported by Takaya et al., 2015. Further limitations of calcium-based assays as dye affinity, ceiling effects of the dynamic range, calcium buffering, and calcium elimination should be mentioned and might affect the inference of the concentration-dependent channel open probability.

The in vitro $\mathrm{EC}_{50}$ value approximately eightfold lower than in psychophysical experiments, which is in line with the literature on other targets, but also with own experience regarding TRPA1, including carvacrol (Schwarz et al., 2017), nitroxyl (Eberhardt et al., 2014), and indirectly with reactive chemical species through aminolevulinic acid (Babes et al., 2016). It can be assumed that redistribution due to diffusion and convection rapidly reduces the injected concentration in vivo, and largely explains the observed differences to the in vitro results.

The high potency reported for JT010 motivated us to use this substance to establish a new pain model with high specificity for TRPA1. The endpoint read used in the prior study dramatically 
underestimates the maximum effect, and by that overestimated the potency. Nevertheless, the determined cellular JT010 $\mathrm{EC}_{50}$ of $0.047 \mu \mathrm{M}$ makes this one of the most potent available human TRPA1 agonists. For comparison, AITC with an $\mathrm{EC}_{50}$ of $8 \mu \mathrm{M}$ might serve as a reference (Bianchi et al., 2012), with other potent and available substances sorted by increasing $\mathrm{EC}_{50}$ as follows: PF-4840154 0.023 $\mu \mathrm{M}$ (Ryckmans et al., 2011); polygodial 0.059 $\mu \mathrm{M}$ (Iwasaki et al., 2009); and ASP7663 $0.51 \mu \mathrm{M}$ (Kojima et al., 2014). Tear gas derivatives with picomolar $\mathrm{EC}_{50}$ values have been reported, including the most potent dibenzo[b,f] [1,4] oxazepine4-carboxylic acid methyl ester (50 pM), but these substances are not commercially available (Gijsen et al., 2010). The specificity of JT010 with respect to other TRP channels was demonstrated for up to $10 \mu \mathrm{M}$ to cover and go beyond the concentration range used in this study.

\section{Quantitative TRPA1 inhibition}

In contrast to our previous study using carvacrol to stimulate TRPA1, the JT010-induced pain could be completely inhibited. In our previous manuscript, $87 \%$ of capsaicin-induced pain was inhibitedbythe TRPV1 antagonistBCTC(4-(3-Chloro-2-pyridinyl)$\mathrm{N}$-[4-(1,1-dimethylethyl)phenyl]-1-piperazinecarboxamide). In comparison, A-967079 showed an even stronger inhibition of JT010-induced pain. This supports the assumption that JT010 $1 \mu \mathrm{M}$ did not activate other targets that are not inhibited by A-967079. Further, and from a clinical and pharmaceutical perspective more importantly, A-967079 can be used to quantify the complete TRPA1-dependent component in a disease or in disease models. This allows evaluation of the significance of TRPA 1 and the potential therapeutic benefit from targeting this receptor.

\section{Limitations}

The insertion pain was positively associated with the pain AUC. The insertion pain varies substantially between injections in every individual and is likely due to parameters, which cannot be easily assessed like the local nerve fiber density. Human volar forearm skin has been chosen mainly for practical reasons. Based on experiments with different animal tissues, we know that response characteristics are largely uniform. Consequently, it can be assumed that the results would be similar in other part of the body. Therefore, acquisition of the insertion pain can be recommended to allow for correction of this confounding factor. A further practical limitation could be considered the required substance handling for the JT010-induced pain model, complicating the usual routine processing using plasticware.

\section{Conclusion}

An easily implementable human TRPA1-specific pain model was established and thoroughly tested. The model is characterized by complete stimulus inhibition by a TRPA1 antagonist, which can serve as reference for further antagonists. Using a TRPA1 antagonist validated in a human model will allow to quantify the TRPA1-dependent component in pathophysiology and disease.

\section{References}

Andersen HH, Lo Vecchio S, Gazerani P, Arendt-Nielsen L (2017) A doseresponse study of topical allyl-isothiocyanate (mustard oil) as human surrogate model of pain, hyperalgesia, and neurogenic inflammation. Pain 158:1723-1732.

Andersson DA, Gentry C, Moss S, Bevan S (2008) Transient receptor potential A1 is a sensory receptor for multiple products of oxidative stress. J Neurosci 28:2485-2494.

Babes A, Sauer SK, Moparthi L, Kichko TI, Neacsu C, Namer B, Filipovic M,
Zygmunt PM, Reeh PW, Fischer MJ (2016) Photosensitization in porphyrias and photodynamic therapy involves TRPA1 and TRPV1. J Neurosci 36:5264-5278.

Bahia PK, Parks TA, Stanford KR, Mitchell DA, Varma S, Stevens SM Jr, Taylor-Clark TE (2016) The exceptionally high reactivity of cys 621 is critical for electrophilic activation of the sensory nerve ion channel TRPA1. J Gen Physiol 147:451-465.

Bandell M, Story GM, Hwang SW, Viswanath V, Eid SR, Petrus MJ, Earley TJ, Patapoutian A (2004) Noxious cold ion channel TRPA1 is activated by pungent compounds and bradykinin. Neuron 41:849-857.

Bautista DM, Jordt SE, Nikai T, Tsuruda PR, Read AJ, Poblete J, Yamoah EN, Basbaum AI, Julius D (2006) TRPA1 mediates the inflammatory actions of environmental irritants and proalgesic agents. Cell 124:1269-1282.

Bautista DM, Pellegrino M, Tsunozaki M (2013) TRPA1: a gatekeeper for inflammation. Annu Rev Physiol 75:181-200.

Bianchi BR, Zhang XF, Reilly RM, Kym PR, Yao BB, Chen J (2012) Species comparison and pharmacological characterization of human, monkey, rat, and mouse TRPA1 channels. J Pharmacol Exp Ther 341:360-368.

Bretag AH (1969) Synthetic interstitial fluid for isolated mammalian tissue. Life Sci 8:319-329.

Chen J, Hackos DH (2015) TRPA1 as a drug target-promise and challenges. Naunyn Schmiedebergs Arch Pharmacol 388:451-463.

Cordero-Morales JF, Gracheva EO, Julius D (2011) Cytoplasmic ankyrin repeats of transient receptor potential A1 (TRPA1) dictate sensitivity to thermal and chemical stimuli. Proc Natl Acad Sci U S A 108:E1184E1191.

Cruz-Orengo L, Dhaka A, Heuermann RJ, Young TJ, Montana MC, Cavanaugh EJ, Kim D, Story GM (2008) Cutaneous nociception evoked by 15-delta PGJ2 via activation of ion channel TRPA1. Mol Pain 4:30.

da Silva Serra I, Husson Z, Bartlett JD, Smith ES (2016) Characterization of cutaneous and articular sensory neurons. Mol Pain 12: 1744806916636387

de la Roche J, Eberhardt MJ, Klinger AB, Stanslowsky N, Wegner F, Koppert W, Reeh PW, Lampert A, Fischer MJ, Leffler A (2013) The molecular basis for species-specific activation of human TRPA1 protein by protons involves poorly conserved residues within transmembrane domains 5 and 6. J Biol Chem 288:20280-20292.

Eberhardt M, Dux M, Namer B, Miljkovic J, Cordasic N, Will C, Kichko TI, de la Roche J, Fischer M, Suárez SA, Bikiel D, Dorsch K, Leffler A, Babes A, Lampert A, Lennerz JK, Jacobi J, Martí MA, Doctorovich F, Högestätt ED, et al (2014) $\mathrm{H} 2 \mathrm{~S}$ and $\mathrm{NO}$ cooperatively regulate vascular tone by activating a neuroendocrine HNO-TRPA1-CGRP signalling pathway. Nat Commun 5:4381.

Eberhardt MJ, Filipovic MR, Leffler A, de la Roche J, Kistner K, Fischer MJ, Fleming T, Zimmermann K, Ivanovic-Burmazovic I, Nawroth PP, Bierhaus A, Reeh PW, Sauer SK (2012) Methylglyoxal activates nociceptors through transient receptor potential channel A1 (TRPA1): a possible mechanism of metabolic neuropathies. J Biol Chem 287:28291-28306.

Everaerts W, Gees M, Alpizar YA, Farre R, Leten C, Apetrei A, Dewachter I, van Leuven F, Vennekens R, De Ridder D, Nilius B, Voets T, Talavera K (2011) The capsaicin receptor TRPV1 is a crucial mediator of the noxious effects of mustard oil. Curr Biol 21:316-321.

Gees M, Alpizar YA, Boonen B, Sanchez A, Everaerts W, Segal A, Xue F, Janssens A, Owsianik G, Nilius B, Voets T, Talavera K (2013) Mechanisms of transient receptor potential vanilloid 1 activation and sensitization by allyl isothiocyanate. Mol Pharmacol 84:325-334.

Geissner E (1995) The pain perception scale-a differentiated and changesensitive scale for assessing chronic and acute pain (in German). Die Rehabilitation 34:XXXV-XLIII.

Gijsen HJ, Berthelot D, Zaja M, Brône B, Geuens I, Mercken M (2010) Analogues of morphanthridine and the tear gas dibenz $[\mathrm{b}, \mathrm{f}][1,4]$ oxazepine (CR) as extremely potent activators of the human transient receptor potential ankyrin 1 (TRPA1) channel. J Med Chem 53:7011-7020.

Hamilton NB, Kolodziejczyk K, Kougioumtzidou E, Attwell D (2016) Proton-gated $\mathrm{Ca}(2+)$-permeable TRP channels damage myelin in conditions mimicking ischaemia. Nature 529:523-527.

Hinman A, Chuang HH, Bautista DM, Julius D (2006) TRP channel activation by reversible covalent modification. Proc Natl Acad Sci U S A 103: $19564-19568$.

Horváth Á, Tékus V, Boros M, Pozsgai G, Botz B, Borbély É, Szolcsányi J, Pintér E, Helyes Z (2016) Transient receptor potential ankyrin 1 
(TRPA1) receptor is involved in chronic arthritis: in vivo study using TRPA1-deficient mice. Arthritis Res Ther 18:6.

Iwasaki Y, Tanabe M, Kayama Y, Abe M, Kashio M, Koizumi K, Okumura Y, Morimitsu Y, Tominaga M, Ozawa Y, Watanabe T (2009) Miogadial and miogatrial with alpha,beta-unsaturated 1,4-dialdehyde moietiesnovel and potent TRPA1 agonists. Life Sci 85:60-69.

Koivisto A, Hukkanen M, Saarnilehto M, Chapman H, Kuokkanen K, Wei H, Viisanen H, Akerman KE, Lindstedt K, Pertovaara A (2012) Inhibiting TRPA1 ion channel reduces loss of cutaneous nerve fiber function in diabetic animals: sustained activation of the TRPA1 channel contributes to the pathogenesis of peripheral diabetic neuropathy. Pharmacol Res 65:149-158.

Kojima R, Nozawa K, Doihara H, Keto Y, Kaku H, Yokoyama T, Itou H (2014) Effects of novel TRPA1 receptor agonist ASP7663 in models of drug-induced constipation and visceral pain. Eur J Pharmacol 723: $288-293$.

Leffler A, Fischer MJ, Rehner D, Kienel S, Kistner K, Sauer SK, Gavva NR, Reeh PW, Nau C (2008) The vanilloid receptor TRPV1 is activated and sensitized by local anesthetics in rodent sensory neurons. J Clin Invest 118:763-776.

Li CL, Li KC, Wu D, Chen Y, Luo H, Zhao JR, Wang SS, Sun MM, Lu YJ, Zhong YQ, Hu XY, Hou R, Zhou BB, Bao L, Xiao HS, Zhang X (2016) Somatosensory neuron types identified by high-coverage single-cell RNA-sequencing and functional heterogeneity. Cell Res 26:83-102.

Macpherson LJ, Hwang SW, Miyamoto T, Dubin AE, Patapoutian A, Story GM (2006) More than cool: promiscuous relationships of menthol and other sensory compounds. Mol Cell Neurosci 32:335-343.

Macpherson LJ, Dubin AE, Evans MJ, Marr F, Schultz PG, Cravatt BF, Patapoutian A (2007) Noxious compounds activate TRPA1 ion channels through covalent modification of cysteines. Nature 445:541-545.

Materazzi S, Fusi C, Benemei S, Pedretti P, Patacchini R, Nilius B, Prenen J, Creminon C, Geppetti P, Nassini R (2012) TRPA1 and TRPV4 mediate paclitaxel-induced peripheral neuropathy in mice via a glutathionesensitive mechanism. Pflugers Arch 463:561-569.

Meents JE, Fischer MJ, McNaughton PA (2016) Agonist-induced sensitisation of the irritant receptor ion channel TRPA1. J Physiol 594:6643-6660.

Meseguer V, Alpizar YA, Luis E, Tajada S, Denlinger B, Fajardo O, Manenschijn JA, Fernández-Peña C, Talavera A, Kichko T, Navia B, Sánchez A, Señarís R, Reeh P, Pérez-García MT, López-López JR, Voets T, Belmonte C, Talavera K, Viana F (2014) TRPAl channels mediate acute neurogenic inflammation and pain produced by bacterial endotoxins. Nat Commun 5:3125.

Mitrovic M, Shahbazian A, Bock E, Pabst MA, Holzer P (2010) Chemonociceptive signalling from the colon is enhanced by mild colitis and blocked by inhibition of transient receptor potential ankyrin 1 channels. Br J Pharmacol 160:1430-1442.

Moilanen LJ, Hämäläinen M, Nummenmaa E, Ilmarinen P, Vuolteenaho K, Nieminen RM, Lehtimäki L, Moilanen E (2015) Monosodium iodoacetateinduced inflammation and joint pain are reduced in TRPAl deficient mice-potential role of TRPA1 in osteoarthritis. Osteoarthritis Cartilage 23:2017-2026.

Nagata K, Duggan A, Kumar G, García-Añoveros J (2005) Nociceptor and hair cell transducer properties of TRPA1, a channel for pain and hearing. J Neurosci 25:4052-4061.

Naik AK, Tandan SK, Dudhgaonkar SP, Jadhav SH, Kataria M, Prakash VR, Kumar D (2006) Role of oxidative stress in pathophysiology of peripheral neuropathy and modulation by $\mathrm{N}$-acetyl-L-cysteine in rats. Eur J Pain 10:573-579.

Obata K, Katsura H, Mizushima T, Yamanaka H, Kobayashi K, Dai Y, Fukuoka T, Tokunaga A, Tominaga M, Noguchi K (2005) TRPAl induced in sensory neurons contributes to cold hyperalgesia after inflammation and nerve injury. J Clin Invest 115:2393-2401.

Roberts K, Shenoy R, Anand P (2011) A novel human volunteer pain model using contact heat evoked potentials (CHEP) following topical skin application of transient receptor potential agonists capsaicin, menthol and cinnamaldehyde. J Clin Neurosci 18:926-932.

Ryckmans T, Aubdool AA, Bodkin JV, Cox P, Brain SD, Dupont T, Fairman E, Hashizume Y, Ishii N, Kato T, Kitching L, Newman J, Omoto K, Rawson D, Strover J (2011) Design and pharmacological evaluation of PF4840154, a non-electrophilic reference agonist of the TrpAl channel. Bioorg Med Chem Lett 21:4857-4859.

Sałat K, Filipek B (2015) Antinociceptive activity of transient receptor potential channel TRPV1, TRPA1, and TRPM8 antagonists in neurogenic and neuropathic pain models in mice. J Zhejiang Univ Sci B 16:167-178.

Schwartz ES, La JH, Scheff NN, Davis BM, Albers KM, Gebhart GF (2013) TRPV1 and TRPA1 antagonists prevent the transition of acute to chronic inflammation and pain in chronic pancreatitis. J Neurosci 33:5603-5611.

Schwarz MG, Namer B, Reeh PW, Fischer MJM (2017) TRPA1 and TRPV1 antagonists do not inhibit human acidosis-induced pain. J Pain 18:526-534.

Takaya J, Mio K, Shiraishi T, Kurokawa T, Otsuka S, Mori Y, Uesugi M (2015) A potent and site-selective agonist of TRPA1. J Am Chem Soc 137:15859-15864.

Takayama Y, Furue H, Tominaga M (2017) 4-isopropylcyclohexanol has potential analgesic effects through the inhibition of anoctamin 1, TRPV1 and TRPA1 channel activities. Sci Rep 7:43132.

Trevisan G, Materazzi S, Fusi C, Altomare A, Aldini G, Lodovici M, Patacchini R, Geppetti P, Nassini R (2013) Novel therapeutic strategy to prevent chemotherapy-induced persistent sensory neuropathy by TRPA1 blockade. Cancer Res 73:3120-3131.

Trevisan G, Benemei S, Materazzi S, De Logu F, De Siena G, Fusi C, Fortes Rossato M, Coppi E, Marone IM, Ferreira J, Geppetti P, Nassini R (2016) TRPA1 mediates trigeminal neuropathic pain in mice downstream of monocytes/macrophages and oxidative stress. Brain 139:1361-1377.

Usoskin D, Furlan A, Islam S, Abdo H, Lönnerberg P, Lou D, Hjerling-Leffler J, Haeggström J, Kharchenko O, Kharchenko PV, Linnarsson S, Ernfors P (2015) Unbiased classification of sensory neuron types by large-scale single-cell RNA sequencing. Nat Neurosci 18:145-153.

Williams EJ (1949) Experimental designs balanced for the estimation of residual effects of treatments. Aust J Sci Res 2:149-168.

Xiao B, Dubin AE, Bursulaya B, Viswanath V, Jegla TJ, Patapoutian A (2008) Identification of transmembrane domain 5 as a critical molecular determinant of menthol sensitivity in mammalian TRPA1 channels. J Neurosci 28:9640-9651. 